\title{
Symmetry Violation in a Superconducting Film with a Square Array of Ferromagnetic Dots.
}

\author{
Serkan Erdin \\ School of Physics 83 Astronomy, University of Minnesota, \\ Minneapolis, MN 55455
}

(October 29, 2018)

\begin{abstract}
We study the equilibrium state of a superconducting film covered with a regular square array of perpendicularly magnetized magnetic dots. The dots induce vortices in the film directly under them and antivortices between the dots. We show that the symmetry of the dot array is spontaneously violated by the vortices. The positions of the vortices and the antivortices depend on the magnetization and the size of the dots.
\end{abstract}

Heterogeneous structures made of ferromagnetic (FM) and superconducting (SC) pieces have attracted a great deal of interest both experimentally [1-7] and theoretically in recent years [8-15]. In contrast to conventional systems, these structures allow the coexistence of ferromagnetism and superconductivity, if FM and SC order parameters are separated in space by a thin insulating layer [8]. Strong interaction of the FM and SC systems not only gives rise to a new class of novel phenomena and physical effects, but also shows the important technological promise of devices whose transport properties can be easily tuned by comparatively weak magnetic fields.

Several realizations of such systems were proposed. However, so far only sub-micron magnetic dots covered by thin SC films have been prepared and studied [1-7]. The experimental samples of FM-SC hybrid systems were prepared by means of electron beam lithography and liftoff techniques [17]. Both in-plane and out-of-plane magnetization was experimentally realized. The dots with magnetization parallel to the plane were fabricated from Co, Ni, Fe, Gd-Co and Sm-Co alloys. For the dots with magnetization perpendicular to the plane, experimenters used Co/Pt multilayers. The FM dots were deposited on thin $\mathrm{SC}$ films made of either $\mathrm{Nb}$ or $\mathrm{Pb}$, whose transition temperatures are around $7-10 \mathrm{~K}$. In these experiments, the effects of commensurability on the transport properties, e.g. the magnetoresistance oscillations and matching anomalies were observed. Further experiments with periodic arrays of magnetic dots or holes [18-21] confirm that the FM dots create and pin vortices.

Motivated by current interest and appeal of dealing with a new class of physical systems, we consider a periodic array of FM dots on SC thin film. We assume that not only the location of dots is regular, but also their magnetic moments are parallel. Such magnetically ordered dot array can be obtained by the field-cooling from magnetic Curie temperature to a temperature below the superconducting transition temperature $T_{s}$. It is worth to mention that zero-field cooling leads to either random dot magnetization for magnetically hard dots, or to an antiferromagnetic ordering if the dots are magnetically soft $[8,11]$.

In our previous work [16], we demonstrated that a single FM dot with sufficiently strong magnetization and large radius generates a vortex or several vortices under itself. In a periodic array of dots they must be compensated by an array of antivortices, since the total flux generated by a vortex is zero. Depending on the dot's magnetization and size, there might exist several vortexantivortex pairs in the array's elementary cell. For simplicity, we consider only one pair. Due to the symmetry of a square array, the vortices are expected to sit at the dot's centers, while the antivortices are located at the centers of the elementary cells. However, symmetric positions may be energetically unfavorable due to the inhomogenaous magnetic field distribution over the film. Thus, the main problem is to find positions of a vortex and an antivortex in an elementary cell.

In this system, the magnetic field induced by inhomogeneous magnetization of the dot array penetrates into a superconductor and generates the $\mathrm{SC}$ vortices, whereas the magnetic field generated by the supercurrents and SC vortices acts on the magnetic system. The method based on London-Maxwell equations to study such a system was developed in [16]. Under the assumption that the sizes of all structures in the problem are larger than the coherence length $\xi$, the London approximation is valid. In section I, we apply the method of the work [16] to periodic FM-SC structures. Next, we apply it to a system in which a square array of thin circular FM dots is placed upon a thin SC film. We conclude with the results and discussion.

\section{ENERGY AND MAGNETIC FIELD IN PERIODIC HETEROGENEOUS FM-SC SYSTEMS}

A periodic heterogeneous FM-SC system such as the regular magnetic dot array upon the SC film or a periodic domain structure in a ferromagnetsuperconductor bilayer can be studied with the method described in [16].The method to study the ferromagnetsuperconductor bilayer in the continuous limit, where the magnetic system's size is larger than effective penetration depth, is given in [22]. In this section, we introduce a more general method which works well both in continuous and discreet limits. In doing so, we assume that the periodic structures of interest are made of very thin 
magnetic textures with the magnetization perpendicular to the plane and SC films. For simplicity, we consider the limit of zero thickness for films with non-zero $2 \mathrm{~d}$ magnetization and density of superconducting electrons. Their energy is calculated over the surface of the SC film. We start with the energy of such 2 d systems:

$$
U=U_{v v}+U_{m v}+U_{m m}
$$

where $U_{v v}$ is the vortex energy, $U_{m v}$ is the energy of magnetization-vortex interaction, and $U_{m m}$ is the magnetic energy. The terms in (1) are as follows (see [16] for details):

$$
\begin{aligned}
U_{v v} & =\int\left[\frac{\varepsilon_{0}}{2 \pi}(\nabla \varphi)^{2}-\frac{\phi_{0}}{16 \pi^{2} \lambda}(\nabla \varphi) \cdot \mathbf{a}^{v}\right] d^{2} r \\
U_{m v} & =-\int\left[\frac{\phi_{0}}{16 \pi^{2} \lambda}\left(\nabla \varphi \cdot \mathbf{a}^{m}-\frac{1}{2} \mathbf{b}^{v} \cdot \mathbf{m}\right] d^{2} r\right. \\
U_{m m} & =-\frac{1}{2} \int \mathbf{b}^{m} \cdot \mathbf{m} d^{2} r
\end{aligned}
$$

where $\varepsilon_{0}=\phi_{0}^{2} / 16 \pi^{2} \lambda, \phi_{0}=h c / 2 e$ is the quantum flux, $\lambda=\lambda_{L}^{2} / d_{s}$ is the effective penetration depth [23], $(\nabla \varphi)$ is the phase gradient induced by the vortex lattice, $\mathbf{a}$ and $\mathbf{b}$ are vector potential and magnetic field at $z=0$, respectively, $\mathbf{m}$ is the $2 \mathrm{~d}$ magnetization. The superscripts $m$ and $v$ indicate the contributions of magnetization and vortices, respectively. In periodic system all these values are periodic functions of coordinates and can be expanded into Fourier-series. For any periodic $2 \mathrm{~d}$ function $\mathbf{f}(\mathbf{r})$, the Fourier expansion is given by

$$
\mathbf{f}(\mathbf{r})=\sum_{\mathbf{G}} \mathbf{f}_{\mathbf{G}} e^{\mathbf{G} \cdot \mathbf{r}} \quad \mathbf{f}_{\mathbf{G}}=\frac{1}{\mathcal{A}} \int \mathbf{f}(\mathbf{r}) e^{\mathbf{G} \cdot \mathbf{r}} d^{2} \mathbf{r} .
$$

where $\mathbf{G}$ are the reciprocal vectors of the periodic system and $\mathcal{A}$ is the unit cell area. Representing all the values $\mathbf{a}, \mathbf{b}, \mathbf{m}$ and $\nabla \varphi$ as Fourier-series (5), plugging them into the integral representation of the energies $(2,3,4)$ and using equality $\int e^{i\left(\mathbf{G}+\mathbf{G}^{\prime}\right) \cdot \mathbf{r}} d^{2} r=\mathcal{A} \delta_{\mathbf{G},-\mathbf{G}^{\prime}}$, we obtain the following expression for the energy per unit cell:

$$
\begin{aligned}
u_{v v} & =\sum_{\mathbf{G}}\left[\frac{\varepsilon_{0}}{2 \pi}\left|(\nabla \varphi)_{\mathbf{G}}\right|^{2}-\frac{\phi_{0}}{16 \pi^{2} \lambda} \nabla \varphi_{\mathbf{G}} \cdot \mathbf{a}_{-\mathbf{G}}^{v}\right], \\
u_{m v} & =-\sum_{\mathbf{G}}\left[\frac{\phi_{0}}{16 \pi^{2} \lambda} \nabla \varphi_{\mathbf{G}} \cdot \mathbf{a}_{-\mathbf{G}}^{m}+\frac{1}{2} \mathbf{b}_{\mathbf{G}}^{v} \cdot \mathbf{m}_{-\mathbf{G}}\right], \\
u_{m m} & =-\frac{1}{2} \sum_{\mathbf{G}} \mathbf{b}_{\mathbf{G}}^{m} \cdot \mathbf{m}_{-\mathbf{G}} .
\end{aligned}
$$

The total vector-potential obeys the London-Pearl equation

$$
\nabla^{2} \mathbf{A}=\frac{1}{\lambda} \mathbf{a} \delta(z)-4 \pi \nabla \times(\mathbf{m} \delta(z))-\frac{\phi_{0}}{2 \pi \lambda}(\nabla \varphi) \delta(z) .
$$

To find the vector-potentials generated by magnetization and vortices, we employ the Fourier-expansions of $\mathbf{a}$, $(\nabla \varphi)$ and $\mathbf{m}$. In this article, we are interested only in the FM subsystems with magnetization in the $z$ direction. The calculations are therefore done along with the Fourier coefficient of $m_{z}$. The Fourier coefficients of the phase gradient are given by (see, for example [23])

$$
(\nabla \varphi)_{\mathbf{G}}=\frac{2 \pi}{\mathcal{A}} \frac{i \mathbf{G} \times \hat{z} F_{\mathbf{G}}}{G^{2}}
$$

where $F_{\mathbf{G}}=\sum_{i} n_{i} e^{i \mathbf{G} \cdot \mathbf{r}_{i}}$ is the structure factor of vortices, $n_{i}$ and $\mathbf{r}_{i}$ indicate the vorticity and the position of the $i$-th vortex respectively. In doing so, we get the Fourier coefficients of the vector- potentials generated by magnetization in the $z$ direction and vortices:

$$
\begin{aligned}
& \mathbf{a}_{\mathbf{G}}^{v}=\frac{\phi_{0}}{\mathcal{A}} \frac{i(\mathbf{G} \times \hat{z}) F_{\mathbf{G}}}{G^{2}(1+2 \lambda G)}, \\
& \mathbf{a}_{\mathbf{G}}^{m}=4 \pi \lambda \frac{i(\mathbf{G} \times \hat{z}) m_{z \mathbf{G}}}{1+2 \lambda G} .
\end{aligned}
$$

Fourier-image of the magnetic field at the interface is $\mathbf{b}_{\mathbf{G}}=i \mathbf{G} \times \mathbf{a}_{\mathbf{G}}$. Substituting the Fourier coefficients of the vector-potentials, magnetic fields, the phase gradient and the magnetization into equations $(6,7,8)$, we find the energy of the periodic structure:

$$
\begin{aligned}
u_{v v} & =\frac{\phi_{0}^{2}}{4 \pi \mathcal{A}^{2}} \sum_{\mathbf{G}} \frac{\left|F_{\mathbf{G}}\right|^{2}}{G(1+2 \lambda G)}, \\
u_{m v} & =-\frac{\phi_{0}}{\mathcal{A}} \sum_{\mathbf{G}} \frac{m_{z \mathbf{G}} F_{-\mathbf{G}}}{1+2 \lambda G}, \\
u_{m m} & =-2 \pi \lambda \sum_{\mathbf{G}} \frac{G^{2}\left|\mathbf{m}_{z \mathbf{G}}\right|^{2}}{1+2 \lambda G} .
\end{aligned}
$$

These equations have rather general character. The only assumptions are that magnetic and superconducting subsystems are 2-dimensional and periodic.

\section{THE SQUARE ARRAY OF FM DOTS}

In this section we consider a square array of the FM dots on a SC film. We assume that criterion of the vortex appearance under a single magnetic dot [16] is satisfied. Then the vortices appear under the FM dots, while antivortices appear outside them. The existence of antivortices is obvious since the energy of a system containing only vortices would grow as the cube of the system's linear size, whereas, in the "neutral" vortex-antivortex system the energy grows as the square of its size. We consider a simple case in which each dot creates only one vortex under them and one antivortex outside (see Fig.1). In this section we find the condition for the appearance of the vortex-antivortex state and their locations minimizing the energy. In order to solve these problems, we follow the theoretical procedure outlined in the previous section. 

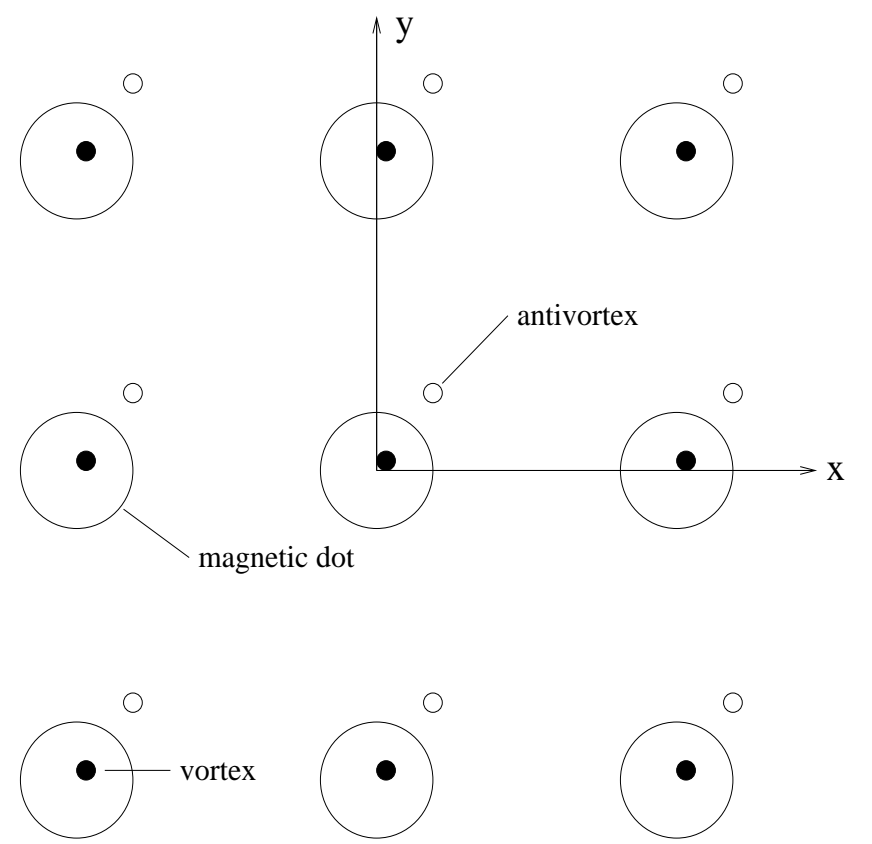

FIG. 1. The top view of the square magnetic dot array is as above. The vortices are confined within the dot's region, while the antivortices appear outside.

The geometry of the FM dots is assumed to be the same as that in the work [16], namely the magnetization of each dot points in positive $z$-direction, they are circular with the radius of each dot equal to $R$. The lattice constant of the square array is denoted as $L$. The SC film and the FM dots are placed at the heights $z=0$ and $z=d>0$, respectively. Later, we transfer to the limit $d=0$ to simplify the results. For a square lattice with the unit cell containing a vortex-antivortex pair, the form factor is:

$$
F_{\mathbf{G}}=\exp \left(i \mathbf{G} \cdot \mathbf{r}_{1}\right)-\exp \left(i \mathbf{G} \cdot \mathbf{r}_{2}\right) .
$$

The set of the reciprocal lattice vectors for the square array is given by equation: $\mathbf{G}=(2 \pi n / L, 2 \pi s / L)$, where $n, s$ are integers; $\mathbf{r}_{1}=x_{1} \hat{x}+y_{1} \hat{y}$ and $\mathbf{r}_{2}=x_{2} \hat{x}+y_{2} \hat{y}$ are the position vectors of the vortex and the antivortex, respectively. In order to find the energy of the system, we need the Fourier-coefficients of the magnetization. Employing the definition of Fourier coefficients for periodic systems in (5), the magnetization of a square array of FM dots reads

$$
m_{z \mathbf{G}}=\frac{2 \pi m R}{L^{2}} \frac{J_{1}(G R)}{G} .
$$

In this result the distance between the dots and SC film $d$ is taken to be zero.

We compute the vortex energy first. Substituting (16) into Eq.(13) gives the energy per unit cell:

$$
u_{v v}=\frac{8 \pi \varepsilon_{0} \lambda}{L^{4}} \sum_{\mathbf{G}}\left[\frac{1}{G(1+2 \lambda G)}-\frac{\cos \left(\mathbf{G} \cdot\left(\mathbf{r}_{2}-\mathbf{r}_{1}\right)\right)}{G(1+2 \lambda G)}\right] .
$$

The cosine term in (18) gives the vortex interaction energy; whereas, the first term is the vortex self energy. In order to extract the logarithmic contribution of the vortex self energy, we split the first term in two parts as $\left(4 \pi \varepsilon_{0} / L^{4}\right) \sum_{\mathbf{G}}\left[1 / G^{2}-1 /\left(G^{2}(1+2 \lambda G)\right)\right]$. The first term is logarithmically divergent and equals $\left(2 \varepsilon_{0} / L^{2}\right) \ln (L / \xi)$. The second term is convergent and will be left in series form. Thus, the vortex energy is found as:

$u_{v v}=\frac{2 \varepsilon_{0}}{L^{2}}\left[\ln \frac{\lambda}{\xi}-\frac{1}{2 \pi} f_{v}(\lambda / L)-\frac{2 \lambda}{L} f_{v v}\left(\lambda / L, \mathbf{r}_{1} / L, \mathbf{r}_{2} / L\right)\right]$.

where the functions $f_{v}(\lambda / L) f_{v v}\left(\lambda / L, \mathbf{r}_{1} / L, \mathbf{r}_{2} / L\right)$ are defined by series:

$$
\begin{aligned}
f_{v} & =\sum_{n, s=-\infty}^{\infty \prime} \frac{1}{\left(n^{2}+s^{2}\right)\left(1+4 \pi \frac{\lambda}{L} \sqrt{n^{2}+s^{2}}\right)}, \\
f_{v v} & =\sum_{n, s=-\infty}^{\infty \prime} \frac{\cos \left(2 \pi\left(n\left(x_{2}-x_{1}\right) / L+s\left(y_{2}-y_{1}\right) / L\right)\right)}{\sqrt{n^{2}+s^{2}}\left(1+4 \pi \frac{\lambda}{L} \sqrt{n^{2}+s^{2}}\right)},
\end{aligned}
$$

where the notation $\sum^{\prime}$ indicates that the term with $n=s=0$ must be omitted.

The magnetization-vortex interaction energy can be obtained by inserting (17) into equation (14). The result is:

$$
u_{m v}=-\frac{m \phi_{0} R}{L^{3}} f_{m v}\left(\lambda / L, R / L, \mathbf{r}_{1} / L, \mathbf{r}_{2} / L\right),
$$

where,

$$
f_{m v}=\sum_{n, s=-\infty}^{\infty \prime} \frac{J_{1}\left(2 \pi \frac{R}{L} \sqrt{n^{2}+s^{2}}\right)\left(\cos \left(2 \pi\left(x_{1} n+y_{1} s\right) / L\right)-\cos \left(2 \pi\left(x_{2} n+y_{2} s\right) / L\right)\right)}{\sqrt{n^{2}+s^{2}}\left(1+4 \pi \frac{\lambda}{L} \sqrt{n^{2}+s^{2}}\right)} .
$$


Now we are in position to calculate the equilibrium locations of the vortices and the antivortices. For this purpose the total energy of the system should be minimized with respect to $\mathbf{r}_{1}$ and $\mathbf{r}_{2}$. For this minimization the magnetization-magnetization interaction can be omitted since it does not depend on the vortex coordinates. Therefore, only the sum

$$
u=u_{v v}+u_{m v}
$$

must be minimized.

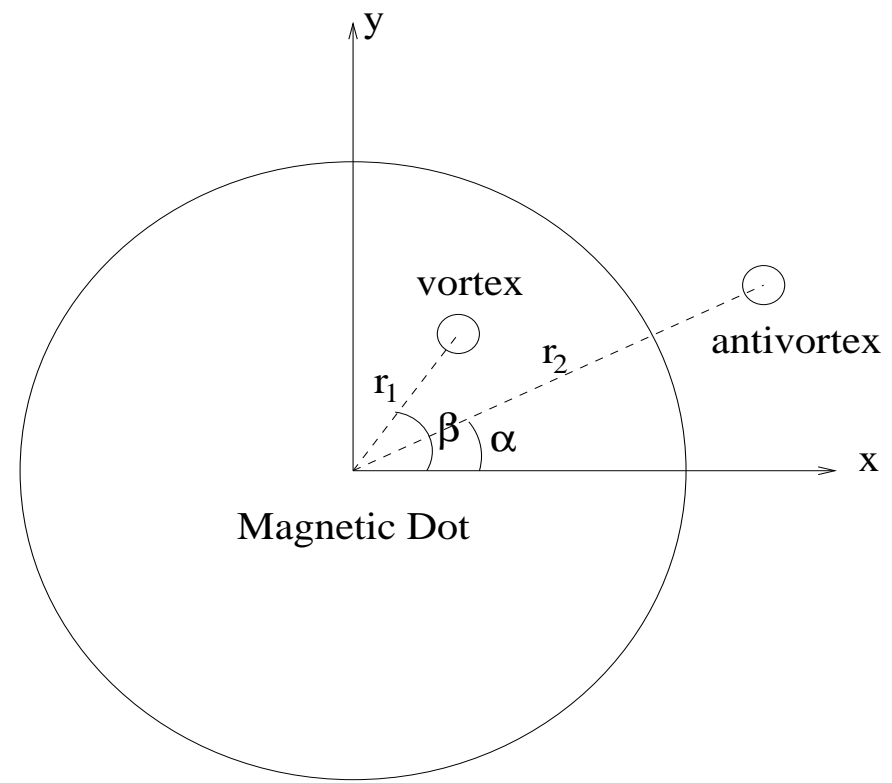

FIG. 2. The test dot with the vortex and the antivortex.

For numerical analysis, it is convenient to rewrite the energy in terms of dimensionless parameters. To this end, we introduce dimensionless variables, namely $\tilde{\lambda}=\lambda / L$, $\tilde{R}=R / L, \tilde{\mathbf{r}}_{1}=\mathbf{r}_{1} / L$ and $\tilde{\mathbf{r}}_{2}=\mathbf{r}_{2} / L$. We also renormalize the total energy per cell $u$ by dividing it by $\varepsilon_{v} / L^{2}$. We place the center of the test dot at the origin, and the positions of the vortices and the antivortices are calculated with respect to that point. Let $\left(\tilde{x}_{1}, \tilde{y}_{1}\right)$ and $\left(\tilde{x}_{2}, \tilde{y}_{2}\right)$ be the respective rescaled coordinates of the vortex and the antivortex relative to the center of the FM dot.

With these modifications, we minimize (23) numerically with respect to $\tilde{\mathbf{r}}_{1}$ and $\tilde{\mathbf{r}}_{2}$ for different values of $m \phi_{0} / \varepsilon_{v}, \tilde{R}$ and $\tilde{\lambda}$. In our numerical calculations, we take $\lambda / \xi=50$. In order for the vortices and the antivortices to appear, the effective energy (see Eq.(23)) must become negative. Using this condition, we find the phase diagrams for the vortex states, which depend on $R / L, R / \lambda$ and $m \phi_{0} / \varepsilon_{v}$. The ratio

$$
\frac{m \phi_{0}}{\varepsilon_{v}}=S g \frac{2 n_{m} d_{m}}{n_{s}(T) d_{s} \ln \kappa(T)}
$$

is the relative strength of the $\mathrm{SC} / \mathrm{FM}$ and controlled by temperature. In Eq.(24), $n_{m}$ is the density of magnetic atoms, $d_{m}$ and $d_{s}$ are the respective thicknesses of magnetic and SC films. $S$ is the value of an elementary spin in the magnet and $g$ is the Lande factor and equal to 2. $n_{s}(T)=n_{s}(T=0)\left(1-T^{2} / T_{s}^{2}\right)$ is the density of super-carriers and $\kappa(T)=\kappa(T=0) / \sqrt{1-T^{2} / T_{s}^{2}}$, where $\kappa(T=0)=\lambda_{L}^{2}(0) / d_{s} \xi(T=0) . \ln \kappa(T)$ varies in the range $3-6$. For typical values of $n_{s}(0)=n_{m}=$ $10^{22} \mathrm{~cm}^{-3}, d_{m}=2 d_{s}, S=2$ and near the SC transition temperature $T / T_{s} \sim 0.95$, the ratio in (24) can be around 30. The curves in Fig.3 indicate when a vortex and an antivortex appear for different $R / L$ ratios. Each curve separates the regions with and without vortex-antivortex pairs. Namely, the region below the curve for particular $R / L$ value represents the region without vortex-and antivortex pair. Above the curve, there exists vortexantivortex pairs. As seen in the figure, the larger the $R / L$ value, the bigger the $m \phi_{0} / \varepsilon_{v}$ ratio. In addition, to create vortex-antivortex pair spontaneously, the minimum value of $m \phi_{0} / \varepsilon_{v}$ increases as $R / \lambda$ ratio decreases for each particular ratio of $R / L$.

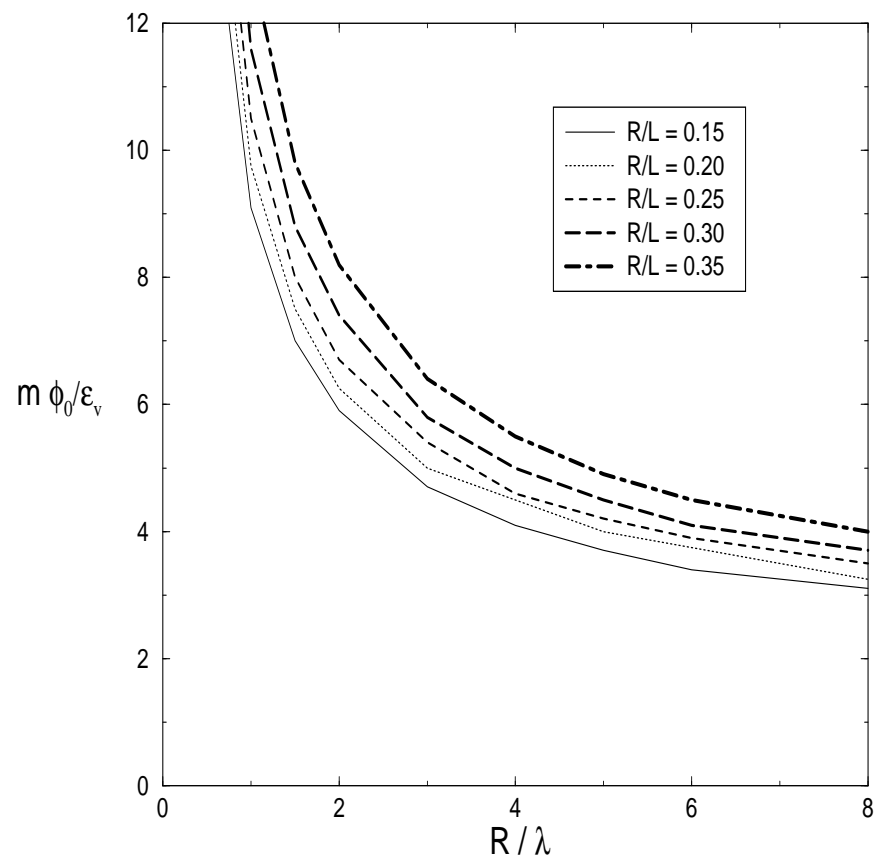

FIG. 3. The phase diagrams of the vortex and the antivortex state for different $R / L$.

Our simulation shows that when the vortices and antivortices appear on the lines which pass through the centers of the dots and make angles $\beta$ and $\alpha$ with the horizontal axis respectively. $\beta$ varies between $3 \pi / 4$ and $\pi$, while $\alpha$ varies between $\pi / 4$ and $\pi / 3$, depending on the size of the dot, the period of the array and the magnetization. It turns out that $\alpha$ gets closer to $\pi / 3$ as $R / L$ increases, whereas $\beta$ approaches $\pi$. As the dots are placed 
further away from one another, the vortex moves closer to the antivortex and the dot's center. Some results are given in the Table I.

TABLE I. The position of vortices and the antivortices for different values of the $R / \lambda$ and $m \phi_{0} / \varepsilon_{v}$. The three columns on the left are input.

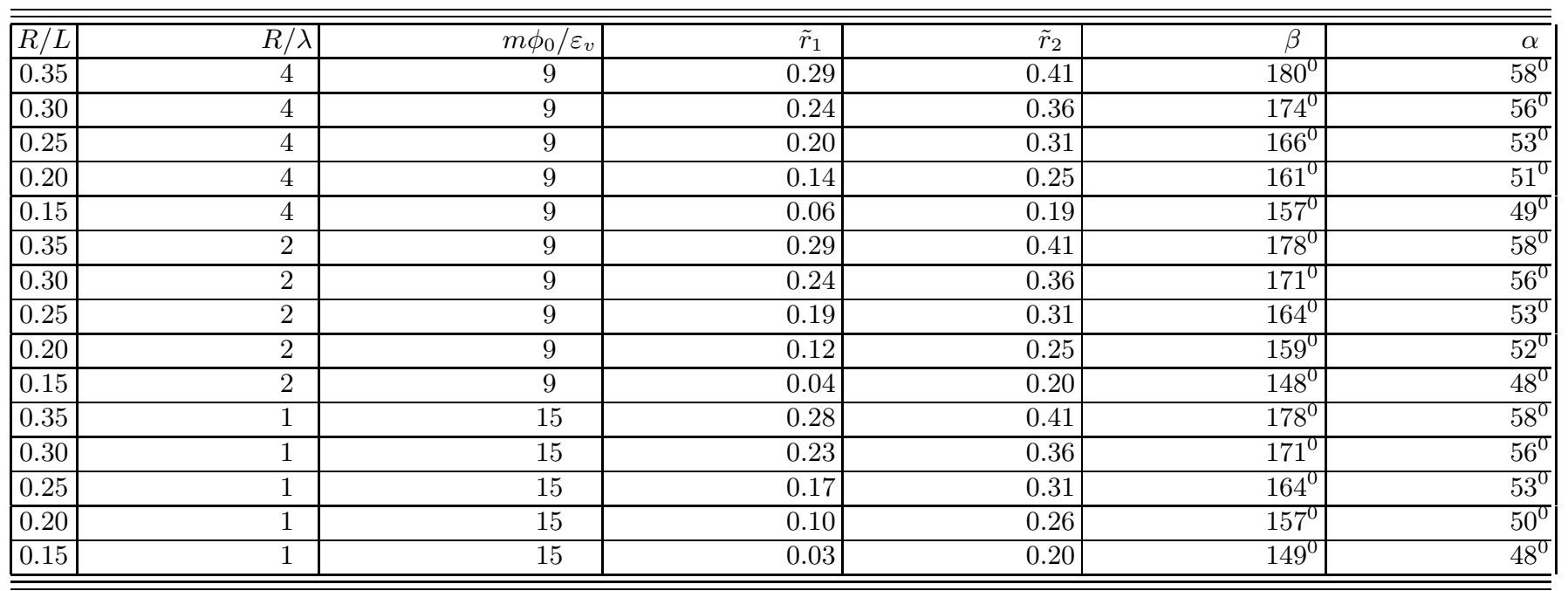

As seen from our results, the symmetry of the ground state is lower than that of the Hamiltonian. The latter has point group $C_{4 d}$ and it has two sets of rotation axis positions: at the centers of the dots and at the centers of squares formed by the dots. The residual symmetry of the ground state is $Z_{2}$, with reflection in all specific lines of squares. This peculiar symmetry violation stems from a highly inhomogeneous magnetic field distribution: the screened magnetic field is logarithmically divergent near the boundaries of the FM dots. As a result, the vortices and the antivortices are dragged into these regions.

\section{CONCLUSIONS}

We considered a periodic array of FM dots on a SC film. In the first section, we presented the formalism which is modified for the case of periodic structures. In this formalism, the problem is formulated as a variational principle which allows us to calculate directly the positions of vortices. We applied this formalism to the case of the square array of the FM dots. We have demonstrated that the dots can generate the vortices under themselves and antivortices at interstitial locations. Thus, the symmetry of the dot array is spontaneously violated by vortices and antivortices. For different values of the size of the dot and the magnetization, we calculated the positions of the vortices and the antivortices and the phase transition curves which separate the regions with and and without vortex-antivortex pairs. The transition is of the first order in our approximation. It turns out that, when the ratio $R / L$ increases, then the minimal value of the ratio $m \phi_{0} / \varepsilon_{v}$ necessary for the spontaneous creation of vortex-antivortex pairs decreases. We assumed that only one vortex-antivortex pair per elementary cell of the array is generated. The problem of many pairs has not yet been solved.

\section{ACKNOWLEDGEMENTS}

This work is mostly done in Physics Department at Texas A\&M University. We would like to thank V.L.Pokrovsky for the statement of the problem, and W.M. Saslow for useful discussions.

[1] J.I. Martin, M. Velez, J. Nogues and I.K. Schuller, Phys. Rev. Lett. 79, 1929 (1997).

[2] D.J. Morgan and J.B. Ketterson Phys. Rev. Lett. 80, 3614 (1998).

[3] Y. Otani, B. Pannetier, J.P. Nozieres and D. Givord, J. Magn. Mag. Mat. 126, 622 (1993).

[4] O. Geoffroy, D. Givord, Y. Otani, B. Pannetier and F. Ossart, J. Magn. Mag. Met. 121, 223 (1993).

[5] Y. Nozaki, Y. Otani, K. Runge, H. Miyajima, B. Pannetier, J.P. Nozieres and G. Fillion, J. Appl. Phys. 79, 8571 (1996). 
[6] M.J. Van Bael, L. Van Look, K. Temst, M. Lange, J. Bekaert, U. May, G. Guntherodt, V.V. Moshchalkov and Y. Bruynseraede, Physica C 332, 12 (2000).

[7] A. Terentiev, D.B. Watkins, L.E. De Long, D.J. Morgan and J.B. Ketterson, Physica C 332, 5 (2000).

[8] I.F. Lyuksyutov and V.L. Pokrovsky, Phys. Rev. Lett. 81, 2344 (1998).

[9] I.F. Lyuksyutov and V.L. Pokrovsky, in Superconducting Superlattices II: Native and Artificial, edited by Ivan Bozovic and Davor Pavuna, SPIE Proceedings Vol. 3480 (SPIE-International Society for Optical Engineering, Bellingham, WA, 1998), p. 230.

[10] I.F. Lyuksyutov and D.G. Naugle, Modern Phys. Lett. B 13, 491 (1999).

[11] Feldman D.E., Lyuksyutov I.F., Pokrovsky V.L. and V.M. Vinokur, Europhys. Lett. 51, 110 (2000).

[12] L.E. Helseth, Phys. Rev. B 66, 104508 (2002).

[13] I.K. Marmorkos, A. Matulis and F.M. Peeters, Phys. Rev. B 53, 2677 (1996).

[14] J.E. Santos, E. Frey and F. Schwabl, Phys. Rev. B 63, 4439 (2001).
[15] M.A. Kayali, Phys. Lett. A 298, 432 (2002).

[16] S. Erdin, A.M. Kayali, I.F. Lyuksyutov, and V.L. Pokrovsky, Phys. Rev. B 66, 014414 (2002).

[17] M.J. Van Bael, K. Temst, V.V. Moshchalkov and Y. Bruynseraede, Phys. Rev. B 59, 14674 (1999).

[18] V.V. Metlushko, M. Baert, R. Jonckheere, V.V. Moshchalkov and Y. Bruynseraede, Solid State Comm. 91, 331 (1994).

[19] M. Baert, V.V. Metlushko, R. Jonckheere, V.V. Moshchalkov and Y. Bruynseraede Phys. Rev. Lett. 74, 3269 (1995).

[20] V.V. Moschalkov, M. Baert, V.V. Metlushko, E. Rosseel, M.J. VanBael, K. Temst, R. Jonckheere and Y. Bruynseraede Phys. Rev. B 54, 7385 (1996).

[21] V.V. Metlushko, L.E. DeLong, M. Baert, E. Rosseel, M.J. VanBael, K. Temst, R. Jonckheere and Y. Bruynseraede, Europhys. Lettl 41,333 (1998).

[22] S.Erdin, I.F. Lyuksyutov, V.L. Pokrovsky and V.M. Vinokur, Phys. Rev. Lett. 88, 017001 (2002).

[23] A.A. Abrikosov, Introduction to the Theory of Metals (North Holland, Amsterdam, 1986). 